\title{
Cost-effectiveness of endovascular thrombectomy in patients with acute ischemic stroke
}

Mattias Aronsson, Josefine Persson, Christian Blomstrand, Per Wester and Lars-Åke Levin

\author{
Linköping University Post Print
}

\section{Tweet}

N.B.: When citing this work, cite the original article.

Original Publication:

Mattias Aronsson, Josefine Persson, Christian Blomstrand, Per Wester and Lars-Åke Levin, Cost-effectiveness of endovascular thrombectomy in patients with acute ischemic stroke, 2016, Neurology, (86), 11, 1053-1059.

http://dx.doi.org/10.1212/WNL.0000000000002439

Copyright: American Academy of Neurology (AAN)

http://www.aan.com/

Postprint available at: Linköping University Electronic Press

http://urn.kb.se/resolve?urn=urn:nbn:se:liu:diva-126429 


\section{Cost-effectiveness of endovascular thrombectomy in patients with acute ischemic stroke}

*Mattias Aronsson, $\mathrm{MSc}^{1}$, Josefine Persson, $\mathrm{MSc}^{2}$, Christian Blomstrand MD, $\mathrm{PhD}^{2}$, Per Wester MD, $\mathrm{PhD}^{3,4}$, Lars-Åke Levin, $\mathrm{PhD}^{1}$

${ }^{1}$ Dept. of Medical and Health Sciences, Linkoping University, Linkoping, Sweden

${ }^{2}$ Dept. of Clinical Neuroscience and Rehabilitation at the Sahlgrenska Academy, University of Gothenburg, Sweden

${ }^{3}$ Dept. of Public Health and Clinical Science, University of Umea, Sweden

${ }^{4}$ Dept Clinical Sciences, Danderyd hospital, Karolinska Institutet, Sweden

Cover title: Endovascular treatment of Ischemic stroke

Search terms: [110] Cost effectiveness/economic, [6] Infarction

Study founding: Supported by grants from the Dental and Pharmaceutical Benefits Agency.

Character count title: 86

Word count abstract: 175

Word count manuscript (incl. tables): 2977

Tables: 2

Figures: $\mathbf{3}$

References: $\mathbf{3 3}$

Supplementary tables: 1

Supplementary figures: $\mathbf{3}$

Statistical analyses were conducted by: Mattias Aronsson

\section{*Corresponding Author:}

Mattias Aronsson, M.Sc.

Linkoping University - Centre for Medical Technology Assessment

Department of Medical and Health Sciences

Linkoping University

SE-581 83 Linkoping, Sweden

Phone: +46 736219021 
Fax: +46 101034995

Email: mattias.aronsson@liu.se

MA: Drafting/revising the manuscript for content, including medical writing for content, Study concept or design and Analysis or interpretation of data.

JP: Drafting/revising the manuscript for content, including medical writing for content and Analysis or interpretation of data.

CB: Drafting/revising the manuscript for content, including medical writing for content and Analysis or interpretation of data.

PW: Drafting/revising the manuscript for content, including medical writing for content and Analysis or interpretation of data.

L-AL: Drafting/revising the manuscript for content, including medical writing for content, Study concept or design and Analysis or interpretation of data.

This study was supported by grants from the Dental and Pharmaceutical Benefits Agency.

Mattias Aronsson reports no disclosures. Josefine Persson reports no disclosures. Dr. Christian Blomstrand reports no disclosures. Dr. Per Wester reports no disclosures. Dr. LarsÅke Levin reports no disclosures. 


\section{ABSTRACT}

\section{Objective}

The aim of this study was to evaluate the cost-effectiveness of adding endovascular thrombectomy to standard care in patients with acute ischemic stroke.

\section{Methods}

The cost-effectiveness analysis of endovascular thrombectomy in patients with acute ischemic stroke was based on a decision-analytic Markov model. Primary outcomes from ESCAPE, EXTEND-IA, MR CLEAN, REVASCAT, and SWIFT PRIME along with data from published studies and registries were used in this analysis. We used a healthcare payer perspective and a lifelong time horizon to estimate costs and effects.

\section{Results}

The model showed that adding thrombectomy with stent retrievers to guideline-based care (including intravenous thrombolysis) resulted in a gain of 0.40 life-years and 0.99 qualityadjusted life-years along with a cost-saving of approximately $\$ 221$ per patient. The sensitivity analysis showed that the results were not sensitive to changes in uncertain parameters or assumptions.

\section{Conclusions}

Adding endovascular treatment to standard care resulted in substantial clinical benefits at low costs. The results were consistent throughout irrespective of whether data from ESCAPE, EXTEND-IA, MR CLEAN, REVASCAT, and SWIFT PRIME were used in this model.

\section{Keywords: Cost-effectiveness, stroke, endovascular treatment, thrombectomy}




\section{INTRODUCTION}

Stroke is a leading cause of serious long-term disability worldwide ${ }^{1-4}$, resulting in decreased quality of life ${ }^{5}$, mortality ${ }^{6}$, increased burden of informal caregivers ${ }^{7}$, and high costs to the society ${ }^{8}$. A primary predictor of the costs associated with strokes is the functional outcome after the stroke ${ }^{9}$. Hence, improved acute care is not only relevant from a medical perspective but also from an economic standpoint.

Intra-arterial treatment has been suggested for patients with proximal occlusions of the major intracranial arteries ${ }^{10}$, which can be administered both pharmacologically and with thrombectomy. The first endovascular randomized controlled trials (IMS- III, MR RESCUE, and SYNTHESIS expansion) ${ }^{11-13}$ without neuroimaging criteria of large-vessel occlusion and using less effective devices were not more effective than using intravenous tissue plasminogen activator. However, the intervention has been refined and five more recently published studies used effective stent retrievers, more rapid door-to-groin puncture, and neuroimaging criteria of proximal vessel occlusion. These randomized studies namely ESCAPE ${ }^{14}$, EXTEND-IA ${ }^{15}$, MR CLEAN ${ }^{10}$, REVASCAT ${ }^{16}$, and SWIFT PRIME ${ }^{17}$ have shown clear benefits of adding endovascular treatment (primarily thrombectomy) to standard care. These studies presented short-term (3-months follow-up) outcome while the long-term effects of the treatment are yet to be studied.

Thus, the aim of this study was to simulate the long-term effects of adding endovascular treatment to standard care in patients with acute ischemic stroke. In addition, the long-term estimates of costs and clinical effects were used to analyze the cost-effectiveness of the treatment. 


\section{MATERIALS AND METHODS}

\section{Analytic approach}

The cost-effectiveness analysis of endovascular treatment in patients with acute ischemic stroke was based on a decision-analytic Markov model. The Markov model is a mathematical model that consists of a number of health states in which the simulated individual can be in and move between. The model is statistically defined as a discrete-time stochastic process consisting of the Markov property. This implies that the future behavior of the model depends only on the current health state of the simulated individual and not on the previous events. Hence, tunnel states were applied in the model to incorporate memory factor and timedependency. Using this simulation model, we estimated the lifelong costs and effects of adding endovascular treatment (mainly thrombectomy) to hypothetical individuals who matched the population of the five randomized studies ${ }^{10,14-17}$ in terms of age and gender distribution. The model approach enabled us to combine the data from short-term randomized controlled trials (RCTs) with that of long-term observational studies in order to make longterm extrapolations. The primary outcome of the model was the cost per gained qualityadjusted life-year (QALY). The analysis had a healthcare payer perspective (including costs for hospital-, primary-, and residential care) and used a lifelong time horizon. A 3\% discount rate was used for both costs and effects throughout the model. In order to make the analysis as consistent as possible, we mainly used cost and population data from a Swedish setting. Both deterministic and probabilistic sensitivity analyses were performed to study the uncertainty of the parameters and assumptions. (Figure e-1 in the supplementary material online describes the core health states and fundamentals of the model.)

\section{Outcome after stroke}

The short-term clinical outcome, defined in this analysis as the outcome during the first 90 days, was for the base-case scenario entirely based on pooled data from the ESCAPE, 
EXTEND-IA, MR CLEAN, REVASCAT, and SWIFT PRIME studies. However, the result of using data from each study separately were also presented. The main outcome data applied from these studies were the modified Rankin Scale (mRS) data obtained after 90 days. The mRS was introduced ${ }^{18}$ in 1957 and later modified into the current version in $1988{ }^{19}$. It is a commonly used tool to measure the degree of dependency or level of global disability after a stroke (0 implies no disability; 6 indicates dead). The global disability score is strongly connected to the amount of costs ${ }^{9}$ and quality of life ${ }^{5}$ in patients, which makes it ideal for our analysis. The randomized, multicenter studies ESCAPE, MR CLEAN, REVASCAT, and SWIFT PRIME collected mRS data after 90 days as the primary endpoint while EXTEND-IA had mRS at 90 days as secondary endpoint. In the MR CLEAN study, 233 patients were assigned to intra-arterial treatment (including thrombolytic agent, thrombectomy, or both) in addition to standard care, while 267 patients in the control group received only the latter. The studies, namely, ESCAPE, EXTEND-IA, REVASCAT, and SWIFT PRIME were all stopped early for ethical reasons after the results from the MR CLEAN study were presented. In these studies, 165, 35, 103, and 98 patients, respectively, were assigned to receive endovascular treatment along with standard care. The outcome for these patients was compared with those of 150, 35, 103, and 98 randomized patients of the control group who received only standard care. Details of the pooled data from the five studies, which were used in the model as the base-case scenario, are presented in Figure 1. The data were pooled with respect to the number of patients with each mRS score in each study and a beta distribution was used to handle the statistical uncertainty.

The short-term outcome after the first 90 days was extrapolated to a lifelong time horizon based on the expected stroke recurrence and mortality in the simulated population (See figure e-1 online for a schematic figure of the extrapolation). The mRS score in the simulated individuals was expected to remain the same until they either experienced a recurrent stroke 
or died (but their quality of life could change for non-stroke related reasons, see Utility weights). This was a conservative assumption based on the modest functional decline observed in the Manhattan Study ${ }^{20}$. The recurrence rate of stroke was obtained from a study with up to 10 years of follow-up data in northern Sweden ${ }^{21}$. After the $10 \mathrm{~h}$ year, the mean recurrence rate (2.4\%) of years 5-10 from the same study was used for the remainder of the simulated patients' life. The risk that a recurrent stroke increased the mRS score in the affected individuals was conservatively assumed to be the same as in the control group in the pooled data of the five thrombectomy studies presented in Figure 1. However, the patients could only transition to health states with the same or with a worse mRS score as compared to the pre-re-stroke health state. For instance, in the case of a recurrent stroke, an individual with mRS score 3 had a 43\% (the added probability to get mRS 0-3 after the index stroke) chance of remaining in the same health state but had a 25, 14 and 18\% risk of transitioning to mRS score 4, 5 and 6 (dead), respectively (see Figure 1).

In the base-case scenario, the long-term annual mortality risk ( $>1$ year) was approximately twice as high as in the general population of Sweden ${ }^{22}$ (HR: 2.0) for both the treatment and the control group. The hazard ratio used was obtained from the mean value of two studies: a study of long-term stroke survival in individuals with atrial fibrillation (HR: 2.1; 95\% CI: $1.8-2.5)^{23}$ and a study of first-ever stroke survivors (HR: 2.0; 95\% CI: $\left.1.7-2.5\right)^{24}$.

\section{Utility weights}

Age-dependent utility weights based on population data were used in the model, ${ }^{25}$ which enabled us to handle the decline in the health-related quality of life caused due to reasons other than stroke. Utility decrements based on the global disability (mRS score) in the simulated individuals after stroke and restroke were used throughout our model. ${ }^{5}$ The observational study used in our model was only available as an abstract; therefore, the 
sensitivity of this parameter was tested by using the other published data that we could identify ${ }^{26}$.

\section{Cost}

The costs for the first year after the initial stroke and re-stroke were obtained from a Swedish study that included data regarding the costs incurred for acute visit, rehabilitation and followup, drugs, home assistance, and residential housing ${ }^{27}$. Cost multipliers (relative costs), as presented by Dawson et al., ${ }^{9}$ were used to distribute the costs according to the mRS score. The same approach was used for determining the long-term stroke-related costs in the simulated patients. The long-term data regarding yearly cost for health care, home assistance, and residential housing, provided in the Swedish cost study, ${ }^{27}$ was distributed accoring to the mRS score (0-3 vs. $4-5$ ) based on Earnshaw et al. ${ }^{28}$. The cost of adding thrombectomy to standard care, \$7 908, was obtained from the university hospitals in Gothenburg, Linköping and Stockholm. All the costs were adjusted according to the inflation rate for the prices in the year 2015 and the exchange rates applicable as of March 1, 2015 (US\$ from Swedish Krona, GBP, and Euro). (The details of the cost data used in the model are presented online in Table e-1.)

\section{Sensitivity analysis}

Two-way sensitivity analyses were conducted to study the importance of uncertain parameters and assumptions (i.e., utility decrements, age of the simulated individuals, cost of adding thrombectomy to standard care, long-term mortality, lifelong time horizon, discount rate and long-term stroke cost). A probabilistic Monte Carlo simulation was used to study the statistical uncertainty in the model. The probability that the treatment is cost-effective was calculated using a threshold of \$10 000 per gained QALY, a conservative threshold compared to what have been suggested in Sweden, the United Kingdom and the Netherlands ${ }^{29}$. 


\section{RESULTS}

On the basis of all the five studies used in this analysis, it was found that the mean mRS score was lower for the group of hypothetical patients who were administered endovascular treatment. Using the model, a slight increase in the mean mRS score of living individuals could be observed over time. Figure 2 shows the proportion of the hypothetical individuals that were alive and the average utility weight in the model. Additionally, the figure shows that the treatment is cost neutral for the healthcare payer after approximately 17 years since stroke. The lifelong model showed that adding endovascular treatment to guideline-based care resulted in a gain of life-years and QALYs along with a low cost (or cost saving), irrespective of using data from any of the five studies separately or using pooled data (Table 2).

\section{Sensitivity analysis}

The reliability of the results were tested with probabilistic analyses. These analyses showed that if pooled data from all five studies was used, there was a probability of $53 \%$ that the intervention was both cost-saving and gained QALYs and a 100\% chance of providing QALYs to a cost below \$10 000 per unit. Further, if the probability of being cost-effective was calculated based on each study (namely, ESCAPE, EXTEND-IA, MR CLEAN, REVASCAT, or SWIFT PRIME) individually, there is 89\%, 90\%, 87\%, 92\%, and 98\% chance, respectively, that adding endovascular treatment to standard care resulted in a cost below \$10 000 per gained QALY. (Acceptability curves and cost-effectiveness planes are shown in figure e-2 and figure e-3 in the supplementary materials.)

The importance of data sources and assumptions used in this model are presented as two-way sensitivity analyses in Figure 3. The figure shows that the results were not sensitive to major changes in the long-term stroke cost, cost of adding thrombectomy, time horizon, the hazard 
ratio of stroke on long-term mortality, mRS scores affecting mortality, age of the simulated individuals, discount rate, or use of other utility weights ${ }^{26}$. Adding endovascular treatment to standard care was still cost-saving or resulted in a low cost per gained QALY.

\section{DISCUSSION}

This study showed that adding endovascular thrombectomy to standard care is dominant compared to standard care alone. This implies that the intervention researched in the ESCAPE, EXTEND-IA, MR CLEAN, REVASCAT, and SWIFT PRIME studies not only provides clinical benefits but also saves cost when using a lifelong perspective.

In contrast to the five studies used in this analysis, three randomized trials (IMS- III, MR RESCUE, and SYNTHESIS expansion) ${ }^{11-13}$ published in 2013 failed to show significant improvement when endovascular treatment was added to standard care. Several possible explanations for this have been previously highlighted ${ }^{10,14}$. For instance, the new types of devices with retrievable stents that were frequently used in the ESCAPE, EXTEND-IA, MR CLEAN, REVASCAT, and SWIFT PRIME studies have shown advantages over the older types of devices ${ }^{30,31}$. In addition, shorter door-to-groin puncture, use of imaging to identify large-vessel occlusion, and excluding patients with a large infarct core and poor collateral circulation were also assumed to have improved the outcomes. The studies chosen in this cost-effectiveness study showed some differences concerning treatments both in control and in intervention groups. However, these studies were included in the analysis because of their similarities (such as retrievable stent retrievers and advanced neuroimaging).

Simulation models, like the one used in this analysis, have limitations when calculating the cost-effectiveness, often due to the need of data regarding lifelong costs and effects. In our study, data from several sources were combined to calculate the long-term results instead of founding the analysis on one single large RCT, prospective collected primary data or a 
published meta-analysis. This was necessary as no RCTs with long enough follow-up to capture all relevant effects were available. The consequence of the absence of long-term RCT data is that the major uncertainties in the model currently are the long-term effects of thrombectomy on the mortality rate and the mRS score. Another related limitation is that all parameter data used in this analysis might not be generalizable to the population where thrombectomy is currently indicated ${ }^{10,14-17}$.

However, waiting for long-term follow-up data from the RCTs before making decisions about an implementation would imply an unacceptable time lag for a promising technology. RCTs with long enough follow-up are, as in this case, rarely available when economic evaluations have to be made. Hence, when policy decisions are required to be made by the decision makers, well-grounded models based on the overall knowledge of the area are useful to be considered. The uncertainties should, as in this analysis, be tested using sensitivity analyses. Furthermore, the model analysis should be updated as soon as data from long-term follow-up studies are available.

The long-term effects of endovascular treatment are still uncertain. In this study, we assumed that there is no relevant improvement in mRS after 90 days. Instead, the mean mRS score is expected to increase slightly annually through the effects on global disability due to recurrent strokes and comorbidities. Increased global disability due to reasons other than stroke was handled using population data from Sweden. This assumption is supported by evidence from published literature ${ }^{20}$.

We used a conservative approach when we assumed that a higher mRS at 90 days after a stroke was not connected with higher long-term mortality. In Figure 3, we provide a scenario where a higher mRS score implied higher mortality ${ }^{26}$. This meant more gain in QALYs but it also meant higher costs as individuals with a high level of dependency lived shorter lives. We also provided a scenario where the hazard ratio for the long-term mortality in the stroke 
population compared to the general population was varied 1 to 4 . The analysis showed that a higher hazard ratio made thrombectomy more costly and less effective but the technology still implied a low cost per gained QALY. Thus, even if the effect from thrombectomy on the long-term mortality is uncertain, it does not have a major impact on the general conclusions from the results of the model.

In this analysis, we used a conservative approach and only estimated costs and effects directly related to the stroke patients. In addition, the estimated costs are based on the general stroke population, which in Sweden, nowadays often suffer a stroke with mild severity. Thus, the median NIHSS in the national Swedish stroke registry Riksstroke ${ }^{32}$ is 3 , while subgroups of patients selected for thrombectomy have considerably higher mRS scores. This means that the health economic gains are undervalued in this pragmatic analysis. Implementing a medical intervention that decreased the global disability and dependency after a stroke onset might also reduce the burden of their relatives. This not only implies gained QALYs but also implies economic gains for the society in terms of fewer hours for providing informal care and thereby less production losses ${ }^{7,33}$. We did not include informal costs in our calculations as we used a healthcare payer perspective. Furthermore, to our knowledge there are no sufficiently detailed data regarding quantity of informal caregivers' support associated to the patients' global disability to be used in the model.

In terms of a cost-effectiveness perspective, it is highly relevant to establish new logistic routines in acute stroke management for implementing the new technology with high priority. This will involve rapid efforts from different parties to identify and strengthen central units to deliver advanced interventional treatment. Furthermore, efficient care chains need to be established between the regional neurovascular centers and the paramedics, community, and specialized non-university hospitals. Urgent requirements will include the mechanisms of handling short-term deficiency of intervention specialists performing thrombectomies and 
dimensioning a potential expansion. Highly specialized clinics performing a large number of thrombectomies as in the recent highly positive studies are probably a prerequisite for the effectiveness of the intervention while a more wide-spread expansion could decrease the possibilities of the requirement of high experience and skills in this advanced technology field. However, it will be a challenge to implement thrombectomy in acute stroke in sparsely populated areas, for instance, in northern Europe. Successful implementation in rural areas of this medical breakthrough will require structured care-chain for telemedicine consultations, “drip-and-ship”, and frequent use of the airborne ambulance.

The results of our study showed that the addition of endovascular treatment, including up-todate stent retrievers, short door-to-groin puncture time, and neuroimaging criteria, to standard care resulted in substantial clinical benefits at low costs. On the basis of this, thrombectomy treatment for an acute ischemic stroke due to proximal artery occlusion seems cost-effective.

\section{DISCLOSURES}

This study was supported by grants from the Dental and Pharmaceutical Benefits Agency.

Mattias Aronsson reports no disclosures. Josefine Persson reports no disclosures. Dr.

Christian Blomstrand reports no disclosures. Dr. Per Wester reports no disclosures. Dr. LarsÅke Levin reports no disclosures.

\section{REFERENCES}

1. Global Burden of Disease Study C. Global, regional, and national incidence, prevalence, and years lived with disability for 301 acute and chronic diseases and injuries in 188 countries, 1990-2013: a systematic analysis for the Global Burden of Disease Study 2013. Lancet Epub 2015 Jun 5.

2. Mozaffarian D, Benjamin EJ, Go AS, et al. Heart disease and stroke statistics-2015 update: a report from the american heart association. Circulation 2015;131:e29-e322. 
3. World health organization. The WHO stroke surveillance. World Health Organization. 29-72004. 2004.

4. Mukherjee D, Patil CG. Epidemiology and the global burden of stroke. World Neurosurg 2011;76:S85-90.

5. Dewilde S, Thijs V, Annemans L, Peeters A, Belgian Stroke Council NP. Quality of Life Decrements after Stroke. Value in Health 2014;17:A331-A331. Abstract.

6. Henriksson KM, Farahmand B, Johansson S, Asberg S, Terent A, Edvardsson N. Survival after stroke--the impact of CHADS2 score and atrial fibrillation. Int J Cardiol 2010;141:18-23.

7. van Exel NJ, Koopmanschap MA, van den Berg B, Brouwer WB, van den Bos GA. Burden of informal caregiving for stroke patients. Identification of caregivers at risk of adverse health effects. Cerebrovasc Dis 2005;19:11-17.

8. Ghatnekar O, Persson U, Glader EL, Terent A. Cost of stroke in Sweden: an incidence estimate. Int J Technol Assess Health Care 2004;20:375-380.

9. Dawson J, Lees JS, Chang TP, et al. Association between disability measures and healthcare costs after initial treatment for acute stroke. Stroke 2007;38:1893-1898.

10. Berkhemer OA, Fransen PS, Beumer D, et al. A randomized trial of intraarterial treatment for acute ischemic stroke. N Engl J Med 2015;372:11-20.

11. Broderick JP, Palesch YY, Demchuk AM, et al. Endovascular therapy after intravenous t-PA versus t-PA alone for stroke. N Engl J Med 2013;368:893-903.

12. Kidwell CS, Jahan R, Gornbein J, et al. A trial of imaging selection and endovascular treatment for ischemic stroke. N Engl J Med 2013;368:914-923.

13. Ciccone A, Valvassori L, Nichelatti M, et al. Endovascular treatment for acute ischemic stroke. N Engl J Med 2013;368:904-913.

14. Goyal M, Demchuk AM, Menon BK, et al. Randomized assessment of rapid endovascular treatment of ischemic stroke. N Engl J Med 2015;372:1019-1030.

15. Campbell BC, Mitchell PJ, Kleinig TJ, et al. Endovascular therapy for ischemic stroke with perfusion-imaging selection. N Engl J Med 2015;372:1009-1018.

16. Jovin TG, Chamorro A, Cobo E, et al. Thrombectomy within 8 hours after symptom onset in ischemic stroke. N Engl J Med 2015;372:2296-2306.

17. Saver JL, Goyal M, Bonafe A, et al. Stent-retriever thrombectomy after intravenous t-PA vs. tPA alone in stroke. N Engl J Med 2015;372:2285-2295.

18. Rankin J. Cerebral vascular accidents in patients over the age of 60. II. Prognosis. Scott Med J 1957;2:200-215.

19. van Swieten JC, Koudstaal PJ, Visser MC, Schouten HJ, van Gijn J. Interobserver agreement for the assessment of handicap in stroke patients. Stroke 1988;19:604-607.

20. Dhamoon MS, Moon YP, Paik MC, et al. Long-term functional recovery after first ischemic stroke: the Northern Manhattan Study. Stroke 2009;40:2805-2811.

21. Pennlert J, Eriksson M, Carlberg B, Wiklund PG. Long-term risk and predictors of recurrent stroke beyond the acute phase. Stroke 2014;45:1839-1841.

22. SCB. Statistics Sweden. Life Tables 2014. http://www.scb.se/. Accessed March 1, 2015.

23. Fang MC, Go AS, Chang Y, et al. Long-term survival after ischemic stroke in patients with atrial fibrillation. Neurology 2014;82:1033-1037.

24. Hankey GJ, Jamrozik K, Broadhurst RJ, et al. Five-year survival after first-ever stroke and related prognostic factors in the Perth Community Stroke Study. Stroke 2000;31:2080-2086.

25. Burstrom K, Johannesson M, Diderichsen F. A comparison of individual and social time tradeoff values for health states in the general population. Health policy 2006;76:359-370.

26. Samsa GP, Reutter RA, Parmigiani G, et al. Performing cost-effectiveness analysis by integrating randomized trial data with a comprehensive decision model: application to treatment of acute ischemic stroke. J Clin Epidemiol 1999;52:259-271.

27. Ghatnekar O. The burden of stroke in Sweden: studies on costs and quality of life based on Riks-Stroke, the Swedish stroke register. [Doctoral thesis.]: Umeå University., 2013.

28. Earnshaw SR, Jackson D, Farkouh R, Schwamm L. Cost-effectiveness of patient selection using penumbral-based MRI for intravenous thrombolysis. Stroke 2009;40:1710-1720. 
29. Cleemput I, Neyt M, Thiry N, et al. Threshold Values for CostEffectiveness in Health Care Health Technology Assessment (HTA). KCE reports 100C (D/2008/10.273/96). Brussels, Belgium: Belgian Health Care Knowledge Centre (KCE), 2008.

30. Nogueira RG, Lutsep HL, Gupta R, et al. Trevo versus Merci retrievers for thrombectomy revascularisation of large vessel occlusions in acute ischaemic stroke (TREVO 2): a randomised trial. Lancet 2012;380:1231-1240.

31. Saver JL, Jahan R, Levy EI, et al. Solitaire flow restoration device versus the Merci Retriever in patients with acute ischaemic stroke (SWIFT): a randomised, parallel-group, non-inferiority trial. Lancet 2012;380:1241-1249.

32. Riks-Stroke: Årsrapport 2013 [Annual report 2013]. Umeå, Sweden; 2014. http://www.riksstroke.org/wp-content/uploads/2014/07/Strokerapport_AKUTTIA3man_LR.pdf. Accessed November 25, 2015.

33. Dewey HM, Thrift AG, Mihalopoulos C, et al. Informal care for stroke survivors: results from the North East Melbourne Stroke Incidence Study (NEMESIS). Stroke 2002;33:1028-1033. 


\section{FIGURE LEGENDS}

Figure 1. Functional outcome at 90 days in included studies and the pooled data set.

The functional outcome according to the score on the modified Rankin Scale. The bars shows the primary outcomes from the ESCAPE, EXTEND-IA, MR CLEAN, REVASCAT, and SWIFT PRIME studies. All studies represent pooled data from the five studies. ET:

Endovascular treatment, SC: Standard care.

Figure 2. Lifelong model results.

The curves shows the estimated survival, mean utility weight and cumulative incremental cost in the model. Cumulative incremental cost represent the net cost of adding endovascular thrombectomy to standard care. ET: Endovascular treatment, SC: Standard care.

Figure 3. Two-way sensitivity analyses.

The bars on the left in the figure show the number of gained QALYs per treated patient and the bars on the right show the incremental cost of intervention. The axis intersects at the result for the base-case scenario. 


\section{TABLES}

Table 1. Summary of important model parameters.

\begin{tabular}{lccc}
\hline Parameter & $\begin{array}{c}\text { Base-case } \\
\text { value }\end{array}$ & Range $^{\mathrm{a}}$ & \\
\hline Age (years) & 67.2 & & Reference \\
Male (\%) & 53.2 & & \\
\hline Time horizon & Lifelong & 5 year-lifelong \\
Discount rate (\%) & 3 & $0-5$ & 21 \\
\hline Stroke recurrence year 1-10 (annual \%) & $0.02-0.06$ & Beta & 23,24 \\
\hline Long-term hazard ratio stroke & 2.0 & $(1.7-2.5)$ &
\end{tabular}

QALY weights

General population

\begin{tabular}{|c|c|c|c|}
\hline $60-69$ & 0.81 & $0.79-0.83$ & 25 \\
\hline $70-79$ & 0.79 & $0.76-0.82$ & \\
\hline 80-88 & 0.73 & $0.68-0.79$ & \\
\hline
\end{tabular}

Stroke decrements

\begin{tabular}{ccl}
\hline $\mathrm{mRS}=0$ & 0.02 & $\operatorname{Exp}(-4.03 \pm 0.59)$ \\
$\mathrm{mRS}=1$ & 0.24 & $\operatorname{Exp}(-1.43 \pm 0.81)$ \\
$\mathrm{mRS}=2$ & 0.25 & $\operatorname{Exp}(-1.38 \pm 0.84)$ \\
$\mathrm{mRS}=3$ & 0.48 & $\operatorname{Exp}(-0.73 \pm 0.91)$ \\
$\mathrm{mRS}=4$ & 0.59 & $\operatorname{Exp}(-0.53 \pm 1.10)$ \\
$\mathrm{mRS}=5$ & 0.84 & $\operatorname{Exp}(-0.17 \pm 1.40)$ \\
\hline a5\% confidence interval was used for probabilistic analyses. mRS: modified Rankin Scale,
\end{tabular}


Table 2. Lifelong results per patient in the base-case scenario.

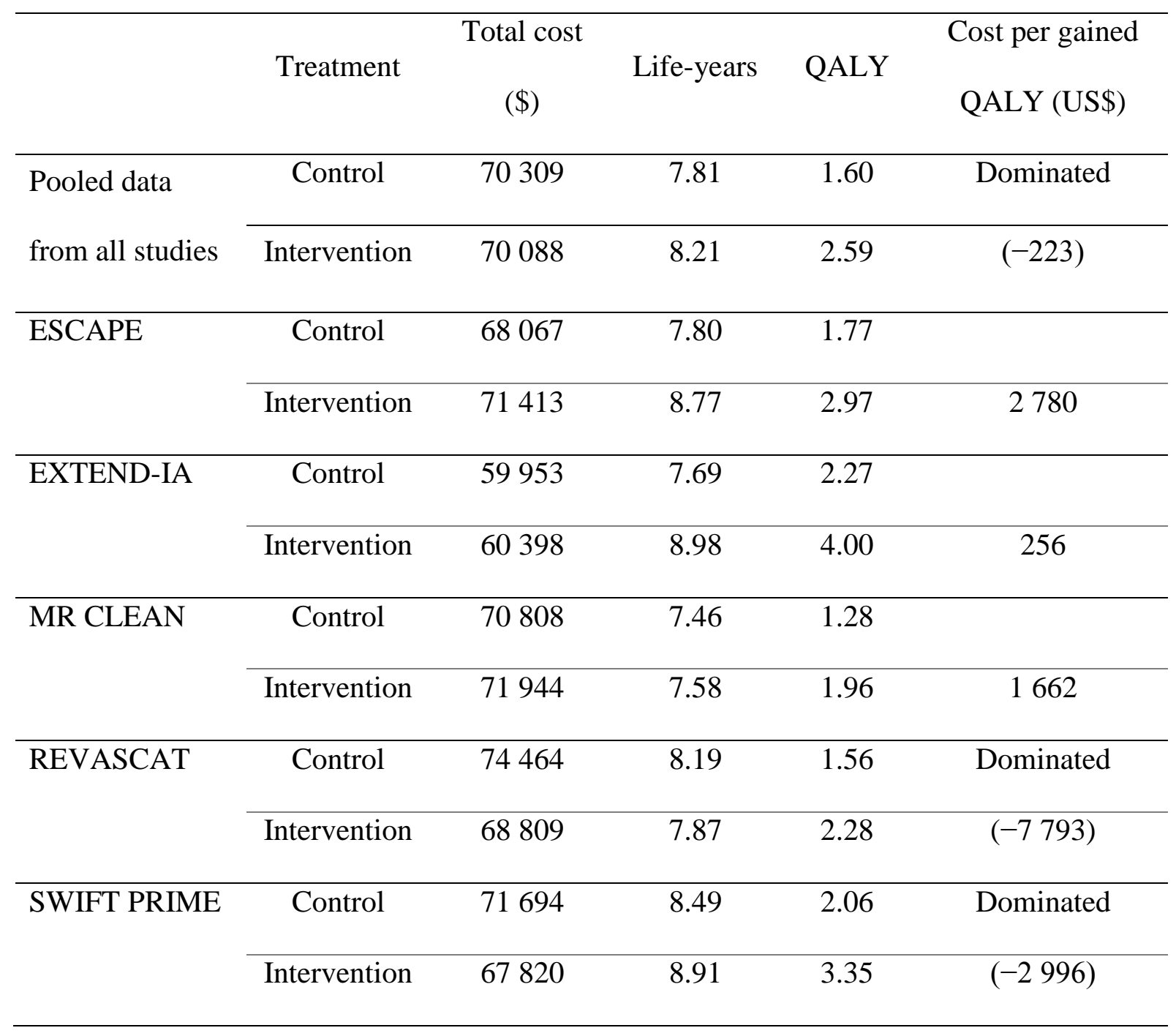




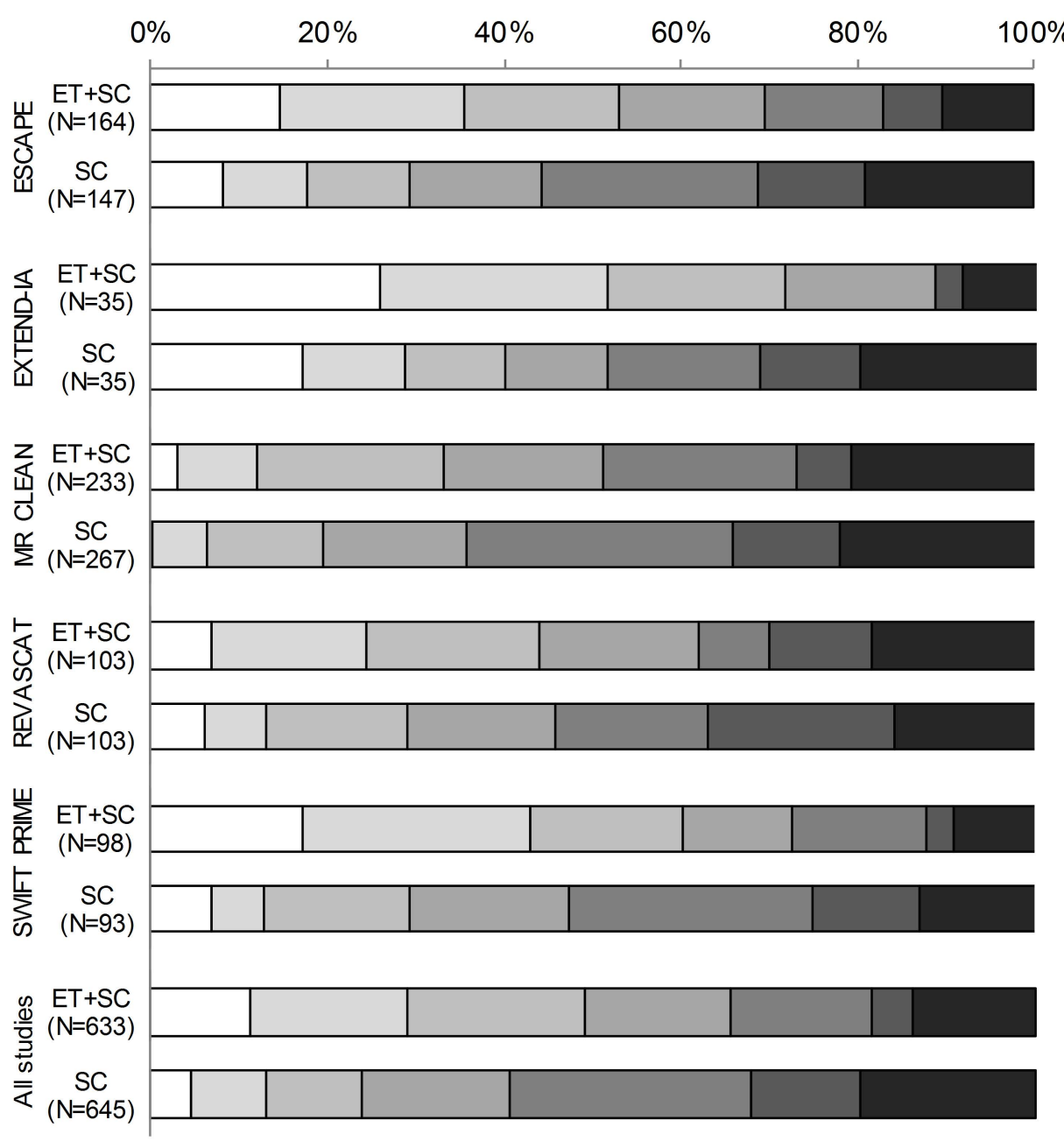

पmRS:0 $\square \mathrm{mRS}: 1 \quad \square \mathrm{mRS}: 2 \quad \square \mathrm{mRS}: 3 \quad \square \mathrm{mRS}: 4 \quad \square \mathrm{mRS}: 5$ 口Dead 


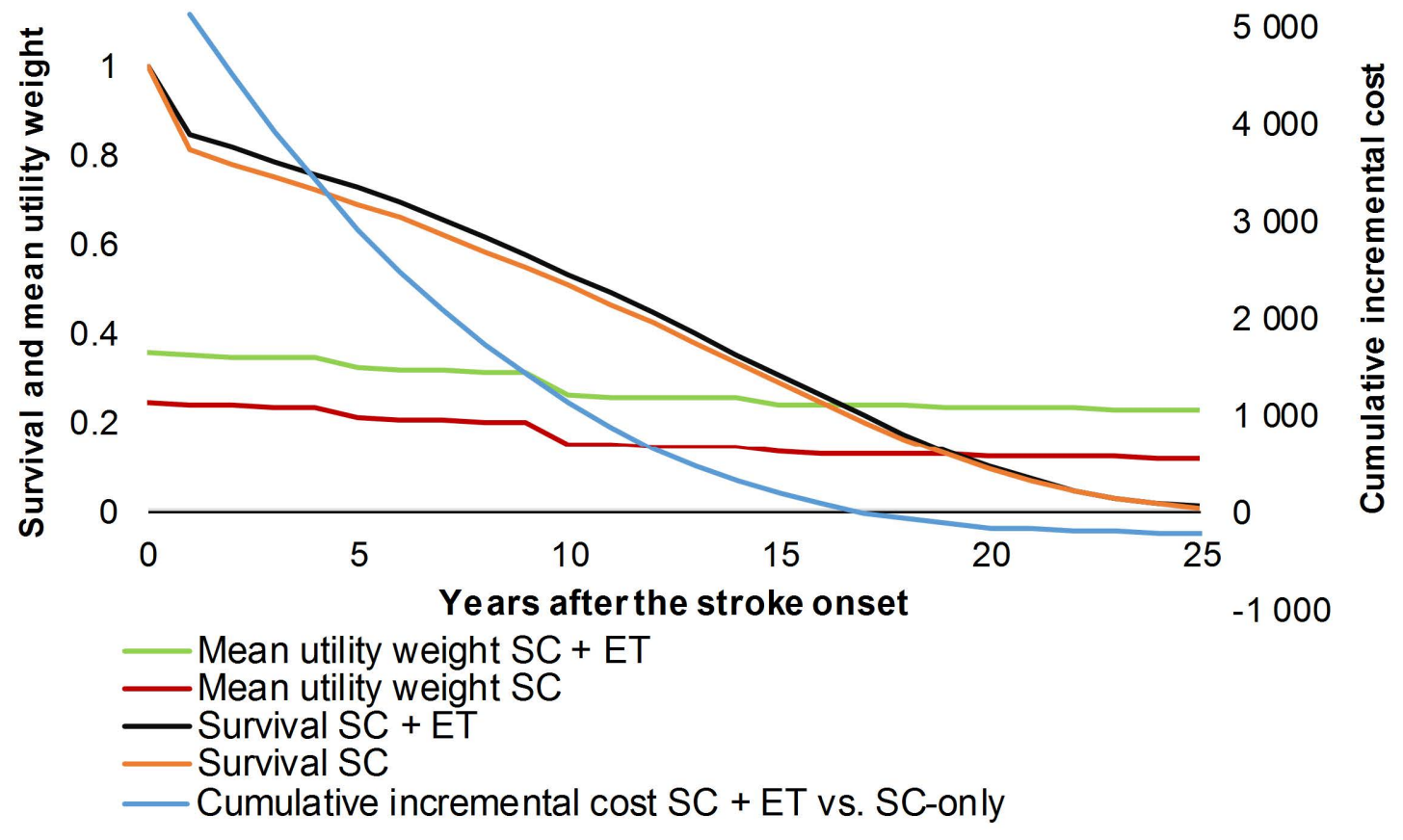


$\square$ Utility-weights from other source [29]

$\square$ Age when enter model (60 - 80 years)

$\square$ Long-term stroke cost (-50\% - +50\%)

$\square$ Cost adding thrombectomy (4 $000-10000$ )

$\square$ Time horizon (5 years)

$\square$ HR long-term stroke mortality (1 - 4)

$\square$ Long-term mortality according to mRS [29]

$\square$ Discount rate $(0-5 \%)$

\section{Incremental cost (US\$)}

